\title{
Investigation of Macro-fin Structure on Heat Transfer Performance of Close-Loop R410A Flashing Spray Cooling
}

\author{
Zhifu Zhou ${ }^{1}$, Renjie $\mathrm{Ji}^{1}$, Bin Chen ${ }^{1 *}$, Panidis Thrassos ${ }^{2}$ \\ ${ }^{1}$ State Key Laboratory of Multiphase Flow in Power Engineering, Xi'an Jiaotong University, \\ Xi'an 710049, China \\ 2 Laboratory of Applied Thermodynamics Mechanical Engineering and Aeronautics \\ Department, University of Patras, 26504, Rio Patras, Greece \\ ${ }^{\star}$ Corresponding author email : chenbin@mail.xjtu.edu.cn
}

\begin{abstract}
The rapid development in performance, integration and miniaturization of electronics and other high power devices cause huge heat flux values. However, transitional cooling methods can hardly meet the requirement of dissipating effectively the high heat flux at the normal working temperature (usually smaller than $75^{\circ} \mathrm{C}$ ). The refrigerants flash evaporation spray cooling with phase change has been considered as one of the most promising technologies of heat dissipation for electronic devices and other high power devices due to its high heat removal capability at relatively low surface temperature, cooling uniformity, non-corrosivity. This study presents an experimental study on the heat transfer performance of the close-loop R410A flashing spray cooling, mainly focusing on the influence of macro-fin structure of cooling surface. The flat, square fin, pyramid fin surfaces and a newly designed tetrahedral fin surface are systematically examined. The results indicate that the newly designed tetrahedral fin surface has superior cooling performance than other fin surfaces.
\end{abstract}

\section{Keywords}

Spray cooling; R410A; heat dissipation; macro-fin surface.

\section{Introduction}

Nowadays, integration and miniaturization are the trend of electronic devices, resulting in chips with high heat flux. It has been predicted that the computer's processor can achieve a heat flux of $300 \mathrm{~W} / \mathrm{cm}^{2}$ to $500 \mathrm{~W} / \mathrm{cm}^{2}$. The heat flux can even reach $1000 \mathrm{~W} / \mathrm{cm}^{2}$ at local hot spots[1]. However, the temperature of the chip needs to be controlled. Once the temperature exceeds $75^{\circ} \mathrm{C}$, the failure rate of electronic components will increase exponentially[2]. Traditional cooling methods (forced convection, pool boiling, heat pipe, jet impact, etc.) have been difficult to meet the requirement of thermal management of electronic equipment. The boiling point of water at atmospheric pressure is $100^{\circ} \mathrm{C}$, and it is not a suitable working fluid. Temperature accumulation reduces the service life of electronic devices and leads to stability decline. Heat dissipation has become a bottleneck restricting the development of high-power equipment. The refrigerant flash spray evaporation spray cooling has high cooling efficiency, low mass flow rate and relatively uniform surface temperature distribution, which is a very promising cooling technology in the field of high-power thermal management[3-5]. The heat transfer mechanism of spray cooling is complex, including droplet impact, film convection and evaporation, nuclear boiling and secondary nucleation, etc.[6]. These heat transfer mechanisms interact with each other, which greatly increases the difficulty of spray cooling research. There have been a lot of studies on spray cooling, in which surface structure improvement is considered to be a very effective method to enhance the performance of spray cooling. 
In the field of macroscopic surface structure, there have been many studies. Silk et al. experimentally studied the influence of macroscopic surface structures such as cubic fin, pyramid fin and long straight fin on heat transfer. The results show that the long straight fin has the greatest influence on heat transfer, and the critical heat flux increases by $55 \%$ compared with the flat surface[7, 8]. Coursey et al. investigated the effect of surface spreading structures, especially straight fins, on heat transfer. The effect of the high-aspect-ratio of the fin is empirically studied. The total surface area and the liquid residence time will vary with the high-aspect-ratio of the fins. The results show that the longer fin has better effect in the single phase heat transfer stage of spray cooling. The enhanced performance of the two phase heat transfer regions in spray cooling is determined by the increased area, the flow rate, the number of channels and the increased thermal conductivity resistance of the fin[9]. Bostanci et al. studied the effect of pyramid fin on critical heat flux at macroscale. It is believed that this structure can retain more liquid on the surface and effectively diffuse the liquid film, so it can delay the emergence of dry point region on the surface with high heat flux, and thus increase the critical heat flux[10]. Xie et al. found that adding fins to the surface does not simply increase the wetting area of the surface, and the layout of the fins plays a decisive role in the cooling performance of the surface. In addition, it has been found that the macroscopic surface has other advantages, such as prolongation of the transition process and shortening of the time that the surface of the heater remains in an extremely high temperature state (film boiling) after the power is turned off[11]. Zhou et al. studied the heat transfer performance of pyramid fin and square fin surfaces. The results show that the surface heat transfer is enhanced due to the increase of the surface wetting area. However, compared with the square fin, the pyramid fin with less increase in wetting area has greater heat transfer enhancement. This indicates that the placement of the fins is more important than increasing the wetting area[12]. The pyramid fin surface is an effective macroscopic structure for enhanced heat transfer. Based on this structure, a more complex macroscopic surface structure called tetrahedral fin surface is designed in this study. The flat, square fin, pyramid fin surfaces and a newly designed tetrahedral fin surface are systematically studied using R410A as the working fluid. The cooling performance of newly designed tetrahedral fin structure is studied compared with other fin surfaces.

\section{Material and Methods}

Experimental system: The schematic diagram of the experimental system is shown in Figure 1. The main components of the system are visual chamber, heater, compressor, condenser, gas-liquid separator and heat exchanger. The experimental working medium is R410A refrigerant, which does not contain any ozone-destroying substances (ODP=0). R410A has good thermophysical properties. The boiling point at atmospheric pressure is $-51.6^{\circ} \mathrm{C}$, and the latent heat of vaporization is as high as $256.7 \mathrm{~kJ} / \mathrm{kg}$. It is an ideal refrigerant for high heat dissipation at low temperature. The compressor compresses the refrigerant into a superheated state at high temperature and pressure. The refrigerant temperature decreases after passing through the condenser and heat exchanger. The high pressure gas can return to the compressor inlet through the upper bypass valve after entering the gas-liquid separator. The high pressure and high temperature gas can be mixed with the gas-liquid two-phase flow before entering the compressor to reduce the risk of liquid shock in the compressor. The liquid refrigerant in the gas-liquid separator accumulates at the bottom. The liquid passes through the flowmeter before entering the visual chamber. The high pressure refrigerant liquid expands and depressurizes through the nozzle and enters the low pressure environment in the chamber. The sudden decrease in pressure causes the refrigerant to overheat, resulting in 
violent crushing and atomization. These fully atomized cryogenic droplets hit the heating surface at the bottom of the chamber. The refrigerant enters the heat exchanger after flowing out of the chamber, and cools the high temperature refrigerant from the condenser again, improving the energy utilization efficiency. The refrigerant enters the compressor and starts the next cycle.

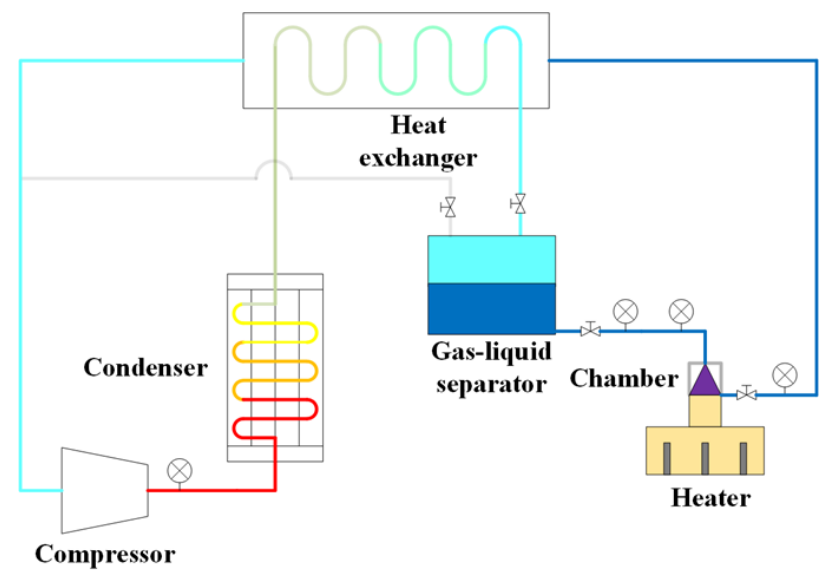

Figure 1. Schematic diagram of experimental system

Experimental facility: The visual chamber diagram used in the experiment is shown in Figure 2 (a). The nozzle diameter is $0.52 \mathrm{~mm}$. The spray distance from the nozzle to the heating surface is adjusted through the screw device. The chamber is made of stainless steel, and the experimental surface and heater are made of copper. The heater is equipped with 10 heating rods with rated power of $150 \mathrm{~W}$, which are connected with the regulated power supply and transformer. The heating power of the heater is controlled by adjusting the voltage input. The experimental surface adapter is shown in Figure 2 (b) and (c). The surface with a diameter of $15 \mathrm{~mm}$ was used to simulate the chip. The surface adapter is placed on the heater and attached using a thermal adhesive (Omega OB-200 Epoxy Adhesive) to eliminate contact gaps to reduce thermal resistance. PEEK (poly-ether-ether-ketone) on the coat of the surface adapter is used to reduce the heat dissipation of the surface adapter to the surrounding. In order to further enhance the insulation effect, a layer of aluminum silicate cotton with a thickness of $10 \mathrm{~mm}$ was wrapped around the whole chamber. Therefore, it can be assumed that the heat transfer from the heater to the surface through the test piece is one-dimensional axial heat conduction, and the heat flux and surface temperature can be calculated by using Fourier law. Two holes with a diameter of $1 \mathrm{~mm}$ are arranged on the side of the surface adapter for placing thermocouples, and the temperature measuring point is located on the central axis. Heat flux $q$, wall temperature $T_{\mathrm{w}}$ and thermal conductivity $h$ are respectively:

$$
\begin{aligned}
& q=\frac{\lambda \Delta T}{d_{1}} \\
& T_{\mathrm{w}}=T_{1}-\frac{q d_{2}}{\lambda} \\
& h=\frac{q}{\Delta T_{\text {sat }}}
\end{aligned}
$$


where $\lambda$ is the thermal conductivity of copper, and $\Delta T$ represents the temperature difference between two thermocouples $T_{1}$ and $T_{2}$, and $d_{1}$ is the distance between two thermocouples while $d_{2}$ is the distance between the surface and the top thermocouple $T_{1}$, and $\Delta T_{\text {sat }}$ is the surface superheat degree, i.e. the temperature difference between saturation temperature $T_{\text {sat }}$ and surface temperature $T_{\mathrm{w}}$.
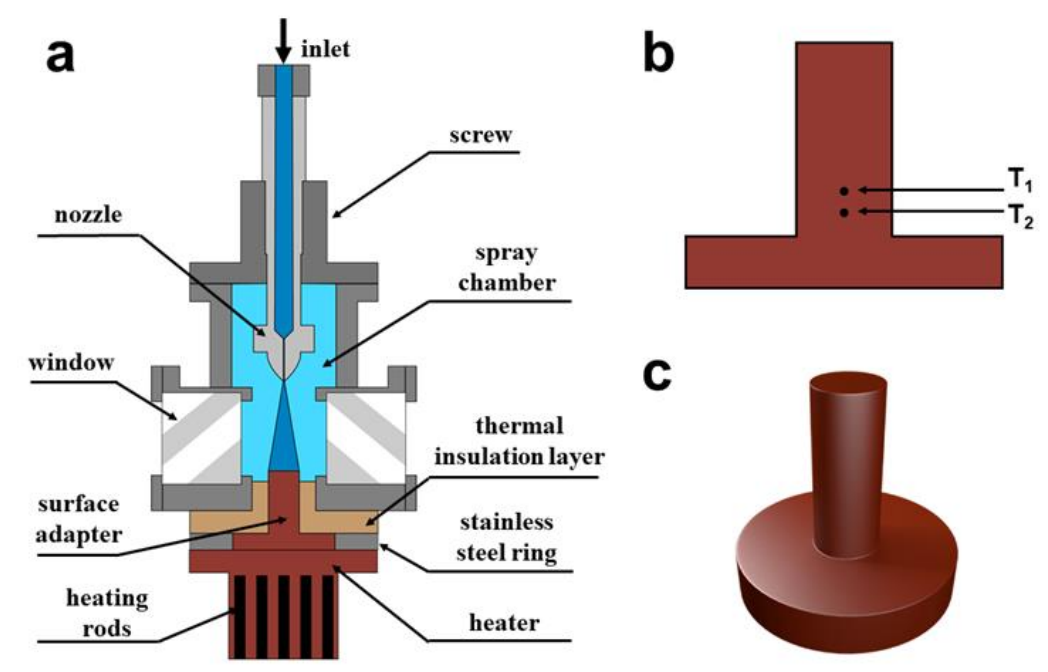

Figure 2. Schematic diagram of visualized chamber and surface adapter :(a) schematic diagram of chamber profile;(b) front view of surface adapter;(c) 3D schematic diagram of the surface adapter

Test surfaces: The macro-fin structure surfaces include square fin, pyramid fin surfaces and a newly designed tetrahedral fin surface, which are processed by milling machine. The flat surface is used as a reference. Physical and confocal laser microscope images of the surfaces are shown in Figure 3.

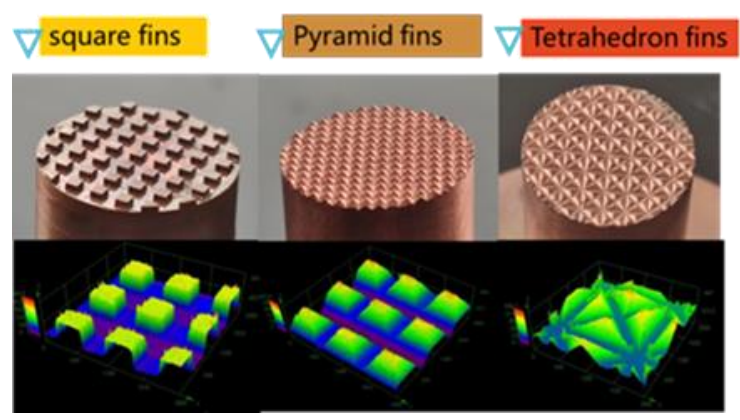

Figure 3. Macro-fin structure surfaces

Experimental procedure: The surface adapter is connected to the heater, and then sealed and installed with the visual chamber, and finally connected to the closed cycle system. Adjust the transformer to input the appropriate power to the heater. The valve on the line regulates the flow rate and the pressure inside the chamber. Mass flow and temperature at the chamber inlet were measured using a Coriolis mass flow meter (ROTAMASS RCCS34). Temperature and pressure signals are collected through thermocouples and pressure sensors. The LabVIEW program on the computer can monitor and record the data, and the sampling rate is $10 \mathrm{~Hz}$. When the temperature measured by the two thermocouples connected to the surface adapter fluctuates within $\pm 0.1^{\circ} \mathrm{C}$ within 10 minutes, the thermal balance is considered to have 
been reached under this working condition. The heat flux and wall superheat are calculated by the temperature of two measuring points and the pressure in the chamber. The experiment involves measuring a range of different conditions, so increasing the power of the heating components is required. Until the experiment under a certain working condition fails to reach a stable thermal balance, or even the temperature rises sharply, it can be considered that the dangerous working condition has been reached, that is, the critical heat flux (CHF) has been reached, and this group of experiments is finished. According to the data of a series of working conditions, the relationship curve between heat flux and wall superheat is drawn to analyze the flash spray cooling performance of the surface.

All experiments were carried out under the same operating conditions, and the detailed information of the experimental conditions is listed in Table 1.

Table 1 - Experimental conditions

\begin{tabular}{c|cc}
\hline & Value & Error \\
\hline Flow rate & $6.1 \mathrm{~g} / \mathrm{s}$ & $\pm 0.2 \mathrm{~g} / \mathrm{s}$ \\
Inlet temperature of the chamber & $28^{\circ} \mathrm{C}$ & $\pm 2^{\circ} \mathrm{C}$ \\
Inlet pressure of the chamber & $2.3 \mathrm{MPa}$ & $\pm 0.06 \mathrm{MPa}$ \\
Outlet pressure of the compressor & $2.33 \mathrm{MPa}$ & $\pm 0.08 \mathrm{MPa}$ \\
Pressure in the chamber & $0.62 \mathrm{MPa}$ & $\pm 0.05 \mathrm{MPa}$ \\
Inlet temperature of the compressor & $-3^{\circ} \mathrm{C}$ & $\pm 2^{\circ} \mathrm{C}$ \\
Outlet temperature of the compressor & $50^{\circ} \mathrm{C}$ & $\pm 4^{\circ} \mathrm{C}$
\end{tabular}

Experimental uncertainty analysis: The errors of heat flux $q$ and heat transfer coefficient $h$ are calculated according to the universal formula of standard error transfer of indirect measurement. The measurement error of the distance between the thermocouples is $\pm 0.1 \mathrm{~mm}$. The diameter of the thermocouple is $1 \mathrm{~mm}$, and the temperature measurement error after calibration is $\pm 1^{\circ} \mathrm{C}$. The measurement error of the mass flowmeter is $\pm 0.15 \%$. The error of the pressure sensor in the measurement range is $\pm 0.05 \mathrm{MPa}$. The material of the surface adapter is copper, and thermal conductivity in a certain temperature range can be considered constant. Its value is $395 \mathrm{~W} /(\mathrm{m} \cdot \mathrm{K})$ and error is $\pm 8 \mathrm{~W} /(\mathrm{m} \cdot \mathrm{K})$. Therefore, the calculation error of heat flux is $\pm 5.79 \%$ at $205.28 \mathrm{~W} / \mathrm{cm}^{2}$. The saturation temperature of the working fluid is estimated by the pressure inside the spray chamber, and the error of heat transfer coefficient is less than $7.9 \%$ when the heat flux is $205.28 \mathrm{~W} / \mathrm{cm}^{2}$.

\section{Results and Discussion}

It is generally considered that the spray cooling curve is divided into three regions. At the beginning, forced convection was the dominant heat transfer mechanism in the single-phase region, and the main behaviors were atomized droplets hitting the wall, liquid film flow and evaporation, etc. With the increase of wall temperature, the heat transfer in the two-phase region is dominated by nuclear boiling. A large number of bubbles were generated at the wall nucleation site and the secondary nucleation site formed after the droplets collided with the liquid film. Boiling can remove a large amount of heat, resulting in a significant increase in heat flux in this region, as shown by an increase in the slope of the curve. As the heat flux continues to increase, the accumulation of bubbles leads to the separation of the working fluid from the wall surface. The heat transfer deteriorates, and some dry patches appear on the surface. The curve also tends to flatten until the critical heat flux arrives. 


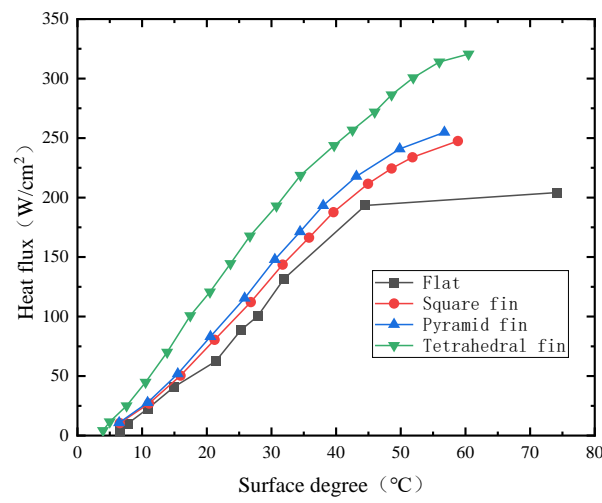

Figure 4. Curves of heat flux and wall superheat of surfaces with different Macro-fin Structure

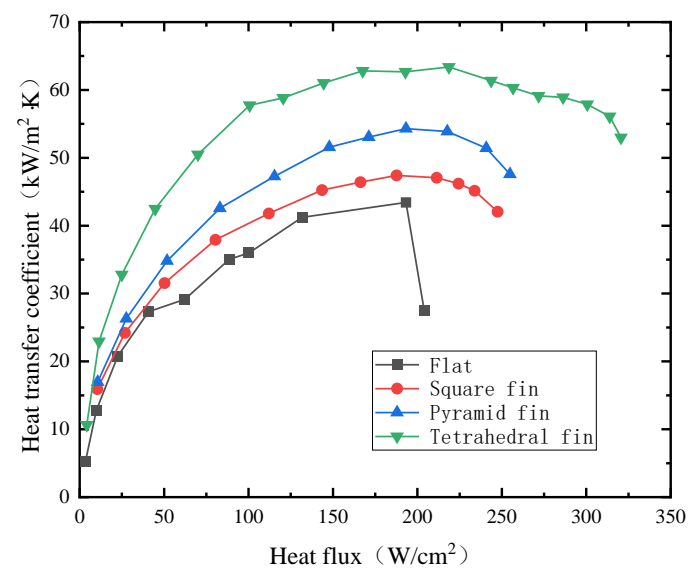

Figure 5. Curves of heat transfer coefficient and heat flux of surfaces with different Macro-fin Structure

Three macroscopic surface structures, square fin, pyramid fin and a newly designed tetrahedral fin, were studied experimentally, and a smooth flat surface was used as a reference. Figure 4 shows the relationship between heat flux and surface superheat on different surfaces. In the single phase region, the cooling performance of each surface is similar. Nuclear boiling occurs on the tetrahedral fin surface at lower wall temperature, and the heat flux of the tetrahedral fin surface is highest among all the surfaces at the same wall temperature. It can be found that the critical heat flux of the square fin and pyramid fin surfaces are both increased to a certain extent compared with the flat surface. The critical heat flux of the tetrahedral fin surface is the highest, reaching $320 \mathrm{~W} / \mathrm{cm}^{2}$, and the superheat of the wall surface is also controlled in a low range. Figure 5 is the curve of heat transfer coefficient of different surfaces. The highest values of heat transfer coefficients on all surfaces occur in the nuclear boiling region. This shows again that nuclear boiling is the most intense heat transfer in the spray cooling process. The highest heat transfer coefficient is $63 \mathrm{~kW} /\left(\mathrm{m}^{2} \cdot \mathrm{K}\right)$ on the tetrahedral fin surface. Therefore, tetrahedral fin surface has the best heat transfer performance, followed by pyramid fin surface, and finned structure significantly increases the critical heat flux value compared with flat surface.

The enhancement of surface heat transfer in macrostructure is firstly caused by the increase of surface wetting area. Compared with the flat surface, the existence of the fin structure will expand the actual surface area, so that the atomized droplets and liquid film have sufficient contact with the surface. At the same time, it greatly increases the number of potential nucleation sites, which is beneficial to nucleation boiling. The regular fin distribution also allows surface channels to facilitate the flow of the accumulated liquid, thus spreading a thinner and 
more uniform film. The critical heat flux is caused by the accumulation of a large number of bubbles at high heat flux, which forms a gas film covering the surface and hinders the heat transfer of droplets on the surface, resulting in further deterioration of heat transfer. This phenomenon can be delayed to some extent by a uniform liquid film and a channel that can quickly guide the liquid. In addition, dry spots always appear first in the place where the temperature is highest, that is, at the base of the fin. On the one hand, the fin structure can separate the gas film formed at the base so that they cannot gather to form large dry patches; on the other hand, the tip part of the fin can still contact the liquid drops for heat exchange. The existence of fin structure plays a significant role in delaying the arrival of critical heat flux and increasing the critical heat flux value. Better heat transfer effect can not be obtained only by increasing the wetting area, and the layout of the fin is more important. The horizontal surface arrangement is superior to the inclined surface arrangement because the droplets impact the wall vertically, while the vertical surface performs worst because it has little contact with the droplets directly impacting. This also explains the poor heat transfer effect on the square fin surface. In addition to the top plane, the fin arrangement has a large number of vertical sides, which has almost no benefit for heat transfer. In the design of macroscopic surface structure, in addition to increasing the wetting area and regular flow channels, more attention should be paid to the layout of the fins.

\section{Conclusions}

By increasing the wetting area and the regular flow channels, the heat transfer effect can be significantly enhanced and the critical heat flux value can be greatly increased. The tetrahedral fin surface has the best cooling performance of all the surfaces. The heat flux is as high as $320 \mathrm{~W} / \mathrm{cm}^{2}$ and the maximum heat transfer coefficient is $63 \mathrm{~kW} /\left(\mathrm{m}^{2} \cdot \mathrm{K}\right)$. Compared with increasing the area and flow channels, the reasonable fin layout is more beneficial to enhance the heat transfer of the surface.

\section{Acknowledgments}

This work was supported by the ministry of science and technology of China with the Key Program for International Science and Technology Cooperation Project (2017YFE0134200)

\section{Nomenclature}

$q \quad$ heat flux $\left[\mathrm{W} / \mathrm{cm}^{2}\right]$

$T_{\mathrm{w}} \quad$ wall temperature $\left[{ }^{\circ} \mathrm{C}\right]$

$h \quad$ heat transfer coefficient $\left[\mathrm{kW} /\left(\mathrm{m}^{2} \cdot \mathrm{K}\right)\right]$

$\lambda \quad$ thermal conductivity $[\mathrm{W} /(\mathrm{m} \cdot \mathrm{K})]$

$\Delta T_{\text {sat }} \quad$ surface superheat degree $\left[{ }^{\circ} \mathrm{C}\right]$

\section{References}

[1] P. Smakulski, S. P., 2016, "A review of the capabilities of high heat flux removal by porous materials, microchannels and spray cooling techniques," Applied Thermal Engineering, 104(5), pp. 636-646.

[2] Rehman, T.-u.-., and Ali, H. M., 2018, "Experimental investigation on paraffin wax integrated with copper foam based heat sinks for electronic components thermal cooling," International Communications in Heat and Mass Transfer(98), pp. 155-162.

[3] Mudawar, I., Bharathan, D., Kelly, K., and Narumanchi, S., 2009, "Two-Phase Spray Cooling of Hybrid Vehicle Electronics," IEEE Transactions on Components and Packaging Technologies, 32(2), pp. 501-512.

[4] Visaria, M., and Mudawar, I., 2009, "Application of Two-Phase Spray Cooling for Thermal Management of Electronic Devices," IEEE Transactions on Components and Packaging Technologies, 32(4), pp. 784-793. 
[5] Hou, Y., Liu, X., Liu, J., Li, M., and Pu, L., 2013, "Experimental study on phase change spray cooling," Experimental Thermal and Fluid Science, 46, pp. 84-88.

[6] Cheng, W.-L., Zhang, W.-W., Chen, H., and Hu, L., 2016, "Spray cooling and flash evaporation cooling: The current development and application," Renewable and Sustainable Energy Reviews, 55, pp. 614-628.

[7] Silk, E. A., Kim, J., and Kiger, K., 2004, "INVESTIGATION OF ENHANCED SURFACE SPRAY COOLING," 2004 ASME International Mechanical Engineering Congress and Exposition, 2.

[8] Silk, E. A., Kim, J., and Kiger, K., 2006, "Spray cooling of enhanced surfaces: Impact of structured surface geometry and spray axis inclination," International Journal of Heat and Mass Transfer, 49(25-26), pp. 4910-4920.

[9] Coursey, J. S., Kim, J., and Kiger, K. T., 2007, "Spray Cooling of High Aspect Ratio Open Microchannels," Journal of Heat Transfer, 129(8).

[10] Bostanci, H., Rini, D. P., Kizito, J. P., Singh, V., Seal, S., and Chow, L. C., 2012, "High heat flux spray cooling with ammonia: Investigation of enhanced surfaces for CHF," International Journal of Heat and Mass Transfer, 55(13-14), pp. 3849-3856.

[11] Xie, J. L., Tan, Y. B., Duan, F., Ranjith, K., Wong, T. N., Toh, K. C., Choo, K. F., and Chan, P. K., 2013, "Study of heat transfer enhancement for structured surfaces in spray cooling," Applied Thermal Engineering, 59(1-2), pp. 464-472.

[12] Zhou, Z.-F., Lin, Y.-K., Tang, H.-L., Fang, Y., Chen, B., and Wang, Y.-C., 2019, "Heat transfer enhancement due to surface modification in the close-loop R410A flash evaporation spray cooling," International Journal of Heat and Mass Transfer, 139, pp. 1047-1055. 